\section{International Scientific Journal Theoretical \& Applied Science}

\author{
p-ISSN: 2308-4944 (print) e-ISSN: 2409-0085 (online) \\ Year: 2017 Issue: $05 \quad$ Volume: 49
}

Published: $3.05 .2017 \quad$ http://T-Science.org
Vadim Anatolyevich Gulyaev Associate Professor of the Department «Equipment and technology of machine-building production»» FSBEI HPE «Togliatti State University», Russia Colmy@tltsu.ru

Nikolay Yuryevich Loginov Associate Professor of the Department «Equipment and technology of machine-building production»» FSBEI HPE «Togliatti State University», Russia

SECTION 7. Mechanics and machine construction.

\title{
AUTOMATED SYSTEM FOR MONITORING THE ROUGHNESS OF THE OUTER SURFACES OF THE OPTICAL-DIGITAL METHOD
}

\author{
Abstract: In this article we describe the system of automated non-contact monitoring of roughness of outer \\ surfaces of parts after machining. \\ Key words: optical-digital method, roughness, roughness control, graphic information processing. \\ Language: Russian \\ Citation: Gulyaev VA, Loginov NY (2017) AUTOMATED SYSTEM FOR MONITORING THE
} ROUGHNESS OF THE OUTER SURFACES OF THE OPTICAL-DIGITAL METHOD. ISJ Theoretical \& Applied Science, 05 (49): 24-27.

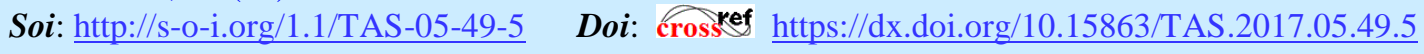

\section{АВТОМАТИЗИРОВАННАЯ СИСТЕМА КОНТРОЛЯ ШЕРОХОВАТОСТИ НАРУЖНЫХ ПОВЕРХНОСТЕЙ ОПТИКО-ЦИФРОВЫМ СПОСОБОМ}

Аннотация: В статье рассматривается система автоматизированного бесконтактного контроля шероховатости наружных поверхностей деталей после механической обработки.

Ключевые слюва: оптико-ичифровой способ, шероховатость, контроль шероховатости, обработка графической информации.

\section{Introduction}

Шероховатость поверхности определяется, как правило, контактным методом с помощью профилометрии микронеровностей, полученных на детали после её механической обработки. Данный метод требует значительных затрат времени и средств. Кроме того, контактный метод не позволяет получать микропрофиль сложных поверхностей (с ребрами, карманами, с изменяющимися в широких пределах кривизнами и т.п.). А также не позволяет проводить экспрессанализ с целью внесения корректировок в ход обработки полученной информации в реальном масштабе времени, что является желательным фактором для повышения качества изделия. Оптические же методы еще более затратны по времени и зависимы от опыта специалиста, проводящего измерения [1]. Так, например, метод визуального контроля с использованием образцов шероховатости наименее трудоемок, но недостаточно точен.

Materials and Methods

Наиболее перспективным является способ, сочетающий преимущества визуального контроля с его высокой производительностью; профилометрический, отличающийся объективностью, основанной на измерительной приборной части и оптический, обладающий высокой точностью воспроизведения поверхности [2, 3]. Таким комбинированным способом является способ, основанный на использовании цифровой фотосъемки полученной после механической обработки поверхности и соответственно алгоритмах, позволяющих производить в реальном масштабе времени компьютерную обработку графической информации, что в итоге позволит установить шероховатость. Для этого, предлагаемый способ, должен быть основан на законах оптической физики, математической статистики и современных информационных технологиях, что даст возможность получать необходимую информацию в производственных условиях. При этом влияние помех, связанных с вибрациями и загрязненностью контролируемой поверхности сведены до минимума, в связи с тем, что время экспозиции при съемке не превышает 


\begin{tabular}{|c|c|c|c|c|c|c|}
\hline Impact Factor: & $\begin{array}{l}\text { ISRA (India) } \\
\text { ISI (Dubai, UAE } \\
\text { GIF (Australia) } \\
\text { JIF }\end{array}$ & $\begin{array}{l}=1.344 \\
=0.829 \\
=0.564 \\
=1.500\end{array}$ & $\begin{array}{l}\text { SIS (USA) } \\
\text { PИНЦ (Russia) } \\
\text { ESJI (KZ) } \\
\text { SJIF (Morocco) }\end{array}$ & $\begin{array}{l}=0.912 \\
=0.234 \\
=3.860 \\
=\mathbf{2 . 0 3 1}\end{array}$ & $\begin{array}{l}\text { ICV (Poland) } \\
\text { PIF (India) } \\
\text { IBI (India) }\end{array}$ & $\begin{array}{l}=6.630 \\
=1.940 \\
=4.260\end{array}$ \\
\hline
\end{tabular}

собственных частот технологического оборудования.

Для достижения этой цели разработана автоматизированная система, основанная на распознавании изображения, которая состоит из оптико-цифрового модуля и персонального компьютера (рисунок 1).

Для функционирования предлагаемой автоматизированной системы используются алгоритмы сравнения текстур изображений при помощи корреляционного критерия сходства по алгоритму сравнения $[4,5,6,7,8,9,10]$.

Основными этапами расчета при функционировании автоматизированной системы для обработки и анализа графической информации являются: предварительная обработка, получение информативных признаков выделенной области изображения, сравнение векторов признаков с набором эталонных признаков.

Для реализации указанных выше задач на всех этапах расчета использовались различные методы и алгоритмы, включая: оптимальнолинейную фильтрацию, вейвлет-преобразования, корелляционные методы, алгоритмы для вычисления оценок.

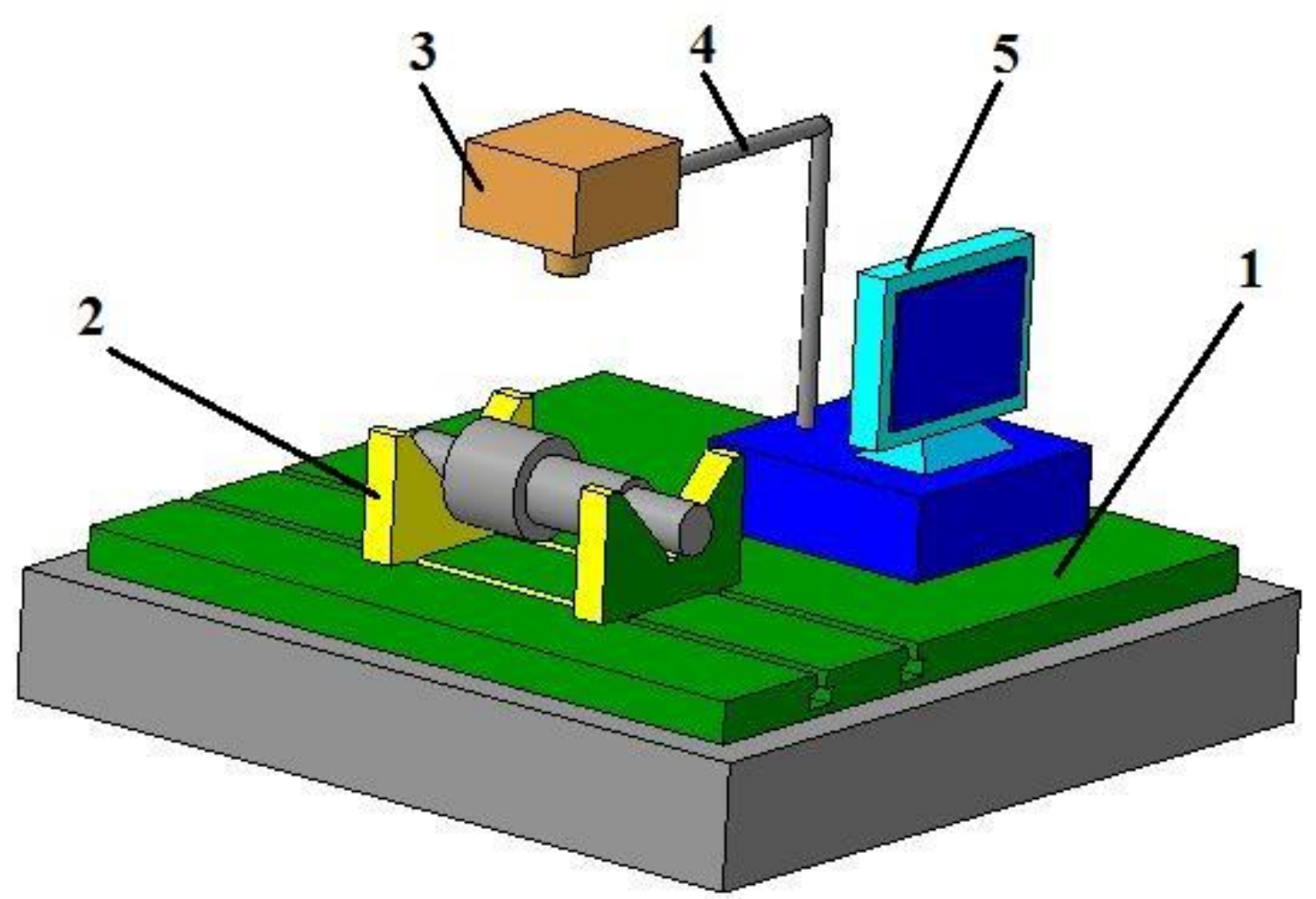

1 - плита;

Рисунок 1 - Оптико-цифровая система определения шероховатости:

2 - призмы;

3 - оптико-цифровой модуль;

4 - штатив;

5 - электронно-вычислительная машина

Текстуры изображений сравниваются по алгоритму работающему на основе корреляционного критерия сходства. Данный алгоритм работает следующим образом.

Эталонный образец шероховатости, представленный в виде матрицы изображения $U_{0}$ имеющий размер $n \times n$, сравнивается с фрагментами снимка входящих в «зону поиска» $\Omega$ имеющими размер $L \times L, L=n+m$. Фрагменты между собой должны перекрываться с шагом $h$, который имеет дискретная решетка $h Z^{2}$ в плоскости $P^{2}$. На данной плоскости задаются наблюдаемые переменные $\left\{u_{0}(x), x=(x, y)\right\}$ на $\mathrm{A}$ или $\{u(x)\}$ на В. Далее выполняется скользящий поиск, при котором текущий фрагмент получается из предыдущего путем сдвига на один 
дискрет. Затем определяется «функция сходства» изображения эталона $\left\{u_{0}(x), x \in \Gamma_{A}\right\}$ и изображения фрагмента подлежащего контролю $\left\{u(x), x \in \Gamma_{B}\right\}$. Данная задача весьма сложная и заключается в поиске функции сходства, которая отвечала бы ряду требований. Основное из которых заключается в том, чтобы она с максимальной точностью и валидностью определяла характеристики текстуры, которые соответствуют изображению фрагмента принятого за эталон, т.е. фактически происходит совмещение сопряженных точек на снимках.

Исходя из того, что необходимо соблюдать условие валидности обнаружения для величины взаимной корреляции $\max \hat{r}(k, l)$ нужно установить порог. Для того чтобы с определенной вероятностью гарантировать сходство контролируемого фрагмента и эталонного фрагмента должно выполняться условие $\max \hat{r}(k, l) \geq r_{\text {nор }}$. Величину порога можно определить функцией распределения коэффициента корреляции для случайных выборок и путем задачи доверительной вероятности принятия решения о действительном сходстве фрагментов.

\section{Conclusion}

Предлагаемый способ универсален. Он позволяет получать качественную оценку шероховатости широкой номенклатуры деталей, получаемых с помощью механической обработки на различном металлорежущем оборудовании. Разработанная методика контроля шероховатости дает возможность также оценивать количественно соответствие поверхности идеальной модели детали (математической или натурной) с реально получаемой. Это в свою очередь создает предпосылки для возможности введения корректировок в работу технологического оборудования в реальном режиме времени с целью повышения качества изделия и достижения соответствия его прообразу.

Предлагаемый оптико-цифровой способ контроля шероховатости наружных поверхностей обладает высокой производительностью, которая в десятки раз превосходит наиболее распространенные контактные и бесконтактные. К тому же, он прост в эксплуатации и не требует специальной подготовки персонала, то есть достаточно обычных навыков при работе с цифровой фотокамерой и компьютерной техникой.

\section{References:}

1. Kozlov AA (2005) Povyshenie kachestva kontrolia zazorov kuzova avtomobilia putem avtomatizatsii protsessa / A. A. Kozlov avtoreferat dissertatsii na soiskanie uchenoi stepeni kandidata tekhnicheskikh nauk / Moskovskii gosudarstvennyi tekhnologicheskii universitet. Toliatti.

2. ZHilin AA, Soldatov AA, Gulyaev VA (2004) Dinamicheskoe nagruzhenie kak metod otsenki dopustimykh vnutrennikh napriazhenii termoobrabotannykh opticheski aktivnykh elementov // Izvestiia Volgogradskogo gosudarstvennogo tekhnicheskogo universiteta 2004. №1. p. 35-36.

3. (2009) Osnovy rascheta i proektirovaniia sistem avtomaticheskogo upravleniia v mashinostroenii: Ucheb. posobie / O. I. Drachev, D. A. Rastorguev, A. A. Soldatov, A. G. Skhirtladze - Staryi Oskol.

4. Soldatov AA, Gulyaev VA, ZHilin AA, Belous DV (2004) Razrabotka avtomatizirovannoi sistemy beskontaktnogo kontrolia geometricheskikh parametrov kuzova avtomobilia // Izvestiia Volgogradskogo gosudarstvennogo tekhnicheskogo universiteta. 2004. №1. p. 46-48.

5. Kozlov AA (2005) Povyshenie kachestva kontrolia zazorov kuzova avtomobilia putem avtomatizatsii protsessa / A. A. Kozlov dissertatsiia na soiskanie uchenoi stepeni kandidata tekhnicheskikh nauk / Toliatti.

6. Kozlov AA (2015) Ekspress-analiz mikrotopografii poverkhnosti pri plakirovanii gibkim instrumentom // V sbornike: Teplofizicheskie i tekhnologicheskie aspekty povysheniia effektivnosti mashinostroitelnogo proizvodstva. Trudy IV mezhdunarodnoi nauchno-tekhnicheskoi konferentsii (Reznikovskie chteniia). 2015. p. 261-264.

7. Kozlov A, Valentiev V (2013) Detection of defects by the Otsu method at control of automobile rear-vew mirrors / Kozlov A., Valentiev V. -V sbornike: PRESSING ISSUES AND PRIORITIES IN DEVELOPMENT OF THE SCIENTIFIC AND TECHNOLOGICAL COMPLEX B\&M Publishing Research and Publishing Center «Colloquium»; Science editor: A. Burkov. San Francisco, California, USA, 2013. p. 109-112. 


\begin{tabular}{l|lrl|l|ll} 
& ISRA (India) & $=\mathbf{1 . 3 4 4}$ & SIS (USA) & $=\mathbf{0 . 9 1 2}$ & ICV (Poland) & $=\mathbf{6 . 6 3 0}$ \\
Impact Factor: & ISI (Dubai, UAE) $=\mathbf{0 . 8 2 9}$ & PUHL (Russia) $=\mathbf{0 . 2 3 4}$ & PIF (India) & $=\mathbf{1 . 9 4 0}$ \\
& GIF (Australia) & $\mathbf{0 . 5 6 4}$ & ESJI (KZ) & $=3.860$ & IBI (India) & $=\mathbf{4 . 2 6 0}$ \\
& JIF & $\mathbf{1 . 5 0 0}$ & SJIF (Morocco) & $=\mathbf{2 . 0 3 1}$ & & \\
\hline
\end{tabular}

8. Gordeev AV, Loginov NI (2015) Optimizatsiia tekhnicheskikh parametrov pri reshenii inzhenernykh zadach // Vektor nauki Toliattinskogo gosudarstvennogo universiteta. 2015. №4(34). p. 25-30.

9. Gruzman IS (2002) Tsifrovaia obrabotka izobrazhenii $\mathrm{v}$ informatsionnykh sistemakh
Ucheb. posobie / I. S. Gruzman, V. S. Kirichuk, V. P. Kosykh - Novosibirsk: Izd-vo NGTU, $352 \mathrm{p}$.

10. Otsu NA (1979) Threshold Selection Method from Gray-Level Histograms, IEEE Transactions on Systems, Man, and Cybernetics, Vol. 9, No. 1, 1979, p. 62-69. 